\title{
The effect of a fibre supplement compared to a healthy diet on body composition, lipids, glucose, insulin and other metabolic syndrome risk factors in overweight and obese individuals
}

\author{
Sebely Pal*, Alireza Khossousi, Colin Binns, Satvinder Dhaliwal and Vanessa Ellis \\ School of Public Health, Curtin Health Innovation Research Institute, ATN Centre for Metabolic Fitness, Curtin University of \\ Technology, GPO Box U1987, Perth, WA, Australia 6485 \\ (Received 3 February 2010 - Revised 20 May 2010 - Accepted 5 July 2010 - First published online 23 August 2010)
}

\section{Abstract}

Optimum levels and types of dietary fibre that provide the greatest beneficial effects on metabolic syndrome risk factors in overweight and obese individuals have yet to be determined in clinical trials. The present parallel design study compared the effects of fibre intake from a healthy diet $v$. a fibre supplement (psyllium) or a healthy diet plus fibre supplement on fasting lipids, glucose, insulin and body composition. Overweight/obese adults were randomised to either control (with placebo), fibre supplement (FIB), healthy eating plus placebo (HLT) or healthy eating plus fibre supplement (HLT-FIB). There was a significant increase in fibre intake in HLT-FIB, HLT and FIB groups up to 59, 31 and $55 \mathrm{~g}$, respectively, at 12 weeks when compared to control $(20 \mathrm{~g})$. Weight, BMI and \% total body fat were significantly reduced in FIB and HLT-FIB groups, with weight and BMI significantly reduced in the HLT group compared with control at 12 weeks. HLT-FIB and HLT groups had significant reductions in TAG and insulin compared with control at 6 and 12 weeks, and in insulin compared with the FIB group at 12 weeks. The HLT-FIB, HLT and FIB groups all had significant reductions in total cholesterol and LDL-cholesterol compared with control after 6 and 12 weeks. The present study demonstrated that simply adding psyllium fibre supplementation to a normal diet was sufficient to obtain beneficial effects in risk factors. However, a high-fibre diet consisting of a psyllium supplement plus fibre from a healthy diet provided the greatest improvements in metabolic syndrome risk factors.

Key words: Fibre: Psyllium: Metabolic syndrome: Obesity: Lipids: Insulin

The growing prevalence of obesity carries with it the increased risk of metabolic syndrome risk factors such as abdominal obesity, atherogenic dyslipidaemia and insulin resistance, which increase the risk of CVD and type 2 diabetes $^{(1)}$. Studies have shown that overweight and obese individuals consume fewer high-fibre foods such as vegetables, fruit, wholegrains and legumes and more fatty and high-energy-dense foods ${ }^{(2)}$. Conversely, epidemiological and cohort studies have indicated that a high-fibre diet is correlated with lower BMI and lower incidence of hyperlipidaemia, CVD and diabetes ${ }^{(2-6)}$.

Dietary fibre is an edible component of all plants and is defined as being resistant to digestion and absorption in the human gut, and usually involves complete or partial fermentation in the large intestine ${ }^{(7,8)}$. There are two main groups of fibre: viscous (water soluble) including pectin, gum, mucilage and psyllium, and non-viscous (water insoluble) including cellulose, hemicellulose and lignin ${ }^{(9)}$. It is estimated that Australian adults consume $18-25 \mathrm{~g}$ of dietary fibre $/ \mathrm{d}^{(10)}$ and US adults consume on an average $15 \mathrm{~g}$ fibre $/ \mathrm{d}^{(8)}$. Present dietary fibre recommendations for adults in Australia and the USA are $25-30 \mathrm{~g} / \mathrm{d}$ to be consumed from fibre-rich sources such as fruit, vegetables, legumes and whole grains ${ }^{(11,12)}$.

Epidemiological and cohort studies have consistently revealed that higher fibre intakes are correlated with lower body weight, BMI, waist circumference ${ }^{(13,14)}$, improved plasma lipid profiles ${ }^{(7,9,15-21)}$ and improved glycaemia and insulinaemia ${ }^{(5)}$, indicating the benefits and risk reduction for the metabolic syndrome, CVD and type 2 diabetes. Lairon et al. ${ }^{(17)}$ found that dietary fibre intakes of $\geq 25 \mathrm{~g} / \mathrm{d}$ was correlated with a reduced risk of a high BMI, cholesterol and hypertension and that $30-35 \mathrm{~g} / \mathrm{d}$ would have even greater protective effects in middleaged adults ${ }^{(17)}$. However, clinical trials have proven to be less conclusive, particularly in relation to weight loss.

Abbreviations: FIB, the supplement group who consumed a fibre supplement with their usual diet; HDL-C, HDL-cholesterol; HLT, a healthy eating group who consumed the placebo with a healthy eating regimen.; HLT-FIB, healthy eating plus fibre supplement group.; LDL-C, LDL-cholesterol; TC, total cholesterol. 
Pittler \& Ernst ${ }^{(22)}$ found a non-significant difference in weight loss when reviewing twenty randomised trials involving guar gum (a soluble fibre). Other intervention studies also reveal no change in body weight ${ }^{(23)}$. However, a review by Howarth et al. ${ }^{(3)}$ concluded that in ad libitum conditions, increasing fibre intake by $14 \mathrm{~g} / \mathrm{d}$ is associated with reduced energy intake and body weight loss. The diversity of the clinical trials may partly explain the inconclusive results as some trials involve increasing fibre intake by consuming a healthy diet of wholegrains, fruit and vegetables $^{(24-27)}$, others involve intake of specific high-fibre foods or enriched foods ${ }^{(28,29)}$ and certain others use fibre supplementation $^{(23,30-32)}$. There are also a few intervention studies addressing the amount of daily fibre intake required to improve metabolic syndrome risk factors in healthy overweight or obese individuals, a population susceptible to the metabolic syndrome. A direct comparison between a group taking fibre supplementation alone and a group taking increased fibre by adopting a healthy eating pattern needs to be undertaken to reveal the effects from these different sources on chronic disease risk factors in the overweight and obese.

Therefore, the aim of the present study was to investigate whether 12 weeks of additional dietary fibre intake in the form of a healthy diet or dietary fibre supplement (psyllium) or a combination of both would induce and sustain improvements to metabolic syndrome risk factors, such as fasting lipids, glucose, insulin and body composition in overweight and obese individuals. We also compared the effects of different amounts of fibre intake (low, moderate and high) on metabolic syndrome risk factors to determine how much dietary fibre intake was most effective.

\section{Materials and methods}

\section{Subjects}

Overweight and obese individuals with a BMI between 25 and $40 \mathrm{~kg} / \mathrm{m}^{2}$ and aged between 18 and 65 years were recruited from the community. Of ninety-four respondents, seventy-two were eligible to participate (thirty-seven men and thirty-five women) and commenced the study. Potential participants were screened by telephone and they attended at Curtin University to assess their suitability for the study, at which time the details of the study were explained. Exclusion criteria included smoking, lipid-lowering medication, use of steroids and other agents that may influence lipid metabolism, use of warfarin, diabetes mellitus, hypo- and hyperthyroidism, cardiovascular events within the last 6 months, psychological unsuitability, major systemic diseases, gastrointestinal problems, proteinuria, liver failure, renal failure, weight flutuations over the past 6 months and vegetarianism. The present study was conducted according to the guidelines laid down in the Declaration of Helsinki and all procedures involving human subjects were approved by the Curtin University Human Research Ethics Committee
(Approval Number HREC 157/2004) with all the participants giving written informed consent.

\section{Methods}

This was a randomised, single-blind, parallel-design study over a 12-week period. The study participants were randomised into one of four groups: the control group ( $n$ 18), who consumed the placebo with their usual diet; the supplement group (FIB, $n$ 18), who consumed a fibre supplement with their usual diet; a healthy eating group (HLT, $n$ 18), who consumed the placebo with a healthy eating regime, and a healthy eating plus fibre supplement group (HLT-FIB, $n$ 18), who consumed the fibre supplement plus a healthy eating regimen. The fibre supplement consisted of $12 \mathrm{~g}$ of psyllium (Metamucil; P\&G, Sydney, NSW, Australia) and the placebo consisted of $12 \mathrm{~g}$ breadcrumbs with flavouring, which provided $1.5 \mathrm{~g}$ of soluble fibre. The breadcrumbs provided an appropriate placebo due to its low energy and fibre content and similarity in texture and appearance to the psyllium supplement. Depending on which group they were allocated to, participants were instructed to take either $12 \mathrm{~g}$ of the fibre supplement or placebo, mixed with $250 \mathrm{ml}$ water, three times daily 5-10 min before breakfast, lunch and dinner. Psyllium is a gel-forming mucilage from the Plantago ovata plant. The bioactive fraction in psyllium is a fibre composed of a highly branched arabinoxylan. Psyllium is a soluble fibre that is gel forming. Psyllium has advantages over other types of soluble fibre because it is less readily fermented and therefore causes less flatulence and abdominal bloating. It was used in the present study as it would be easily tolerated and is readily marketed worldwide under the trade name Metamucil.

The subjects attended a briefing session on how to complete food records and comply with the study protocol. The dietary intake over the course of the trial was monitored through the completion of $3 \mathrm{~d}$ weighed food diaries at baseline, 6 and 12 weeks. Ad libitum healthy eating plans, as designed by a registered dietitian, were prescribed for the HLT and HLT-FIB groups for the 12 weeks, which included healthy meals and snacks based on the Dietary Guidelines for Australian Adults ${ }^{(11)}$. These study participants were asked to record their food and drink intake for 3 d every 3 weeks to monitor the adherence to the guidelines ${ }^{(11)}$ and were given feedback and strategies if necessary. These participants were tightly monitored to ensure their compliance to the dietary guidelines. The participants in the control and FIB groups were asked to maintain their usual dietary intake for the duration of the study. To monitor compliance, all the participants were provided with a measuring cup to measure either the fibre supplement or placebo, they were required to complete a diary to record their supplement consumption and they were asked to return the empty and non-empty containers of the supplements at their 6- and 12-week visits. 


\section{Assessments}

The participants attended at Curtin University, after a 10-12 h fast, for baseline measurements. Subsequent to a mandatory rest period of approximately $30 \mathrm{~min}$, fasting blood samples $(5 \mathrm{ml})$ were drawn by venepuncture. The collection of fasting blood samples was repeated at 6 and 12 weeks. Blood samples were centrifuged at $2500 \mathrm{rpm}$ at $4^{\circ} \mathrm{C}$ for $10 \mathrm{~min}$ using a Hettich Rottina $48 \mathrm{R}$ centrifuge (Tuttlingen, Germany). Serum and plasma were collected to measure circulating levels of TAG, total cholesterol (TC), HDL, LDL, insulin and blood glucose. Serum and plasma samples were stored at $-80^{\circ} \mathrm{C}$ and analysed within 4-6 weeks of collection.

Measures of body weight, height, waist and hip circumference were also undertaken at baseline, 6 and 12 weeks.
Body weight (UM-018 Digital Scales, Tanita Corporation, Tokyo, Japan) was recorded in light clothing without shoes. Height was measured to the nearest $0 \cdot 1 \mathrm{~cm}$ using a stadiometer (26SM $200 \mathrm{~cm}$; SECA, Hamburg, Germany) without shoes. Waist circumference was measured in the standing position at the narrowest area between the lateral lower rib and the iliac crest. Hip measurement was taken at the largest circumference of the lower abdomen. Total body fat and lean mass as well as percentage fat were assessed by whole body dual-energy X-ray absorptiometry (Lunar Prodigy; Lunar, Madison, WI, USA) at baseline and at 12 weeks. The dual-energy X-ray absorptiometry machine was calibrated and a phantom scan was undertaken daily.

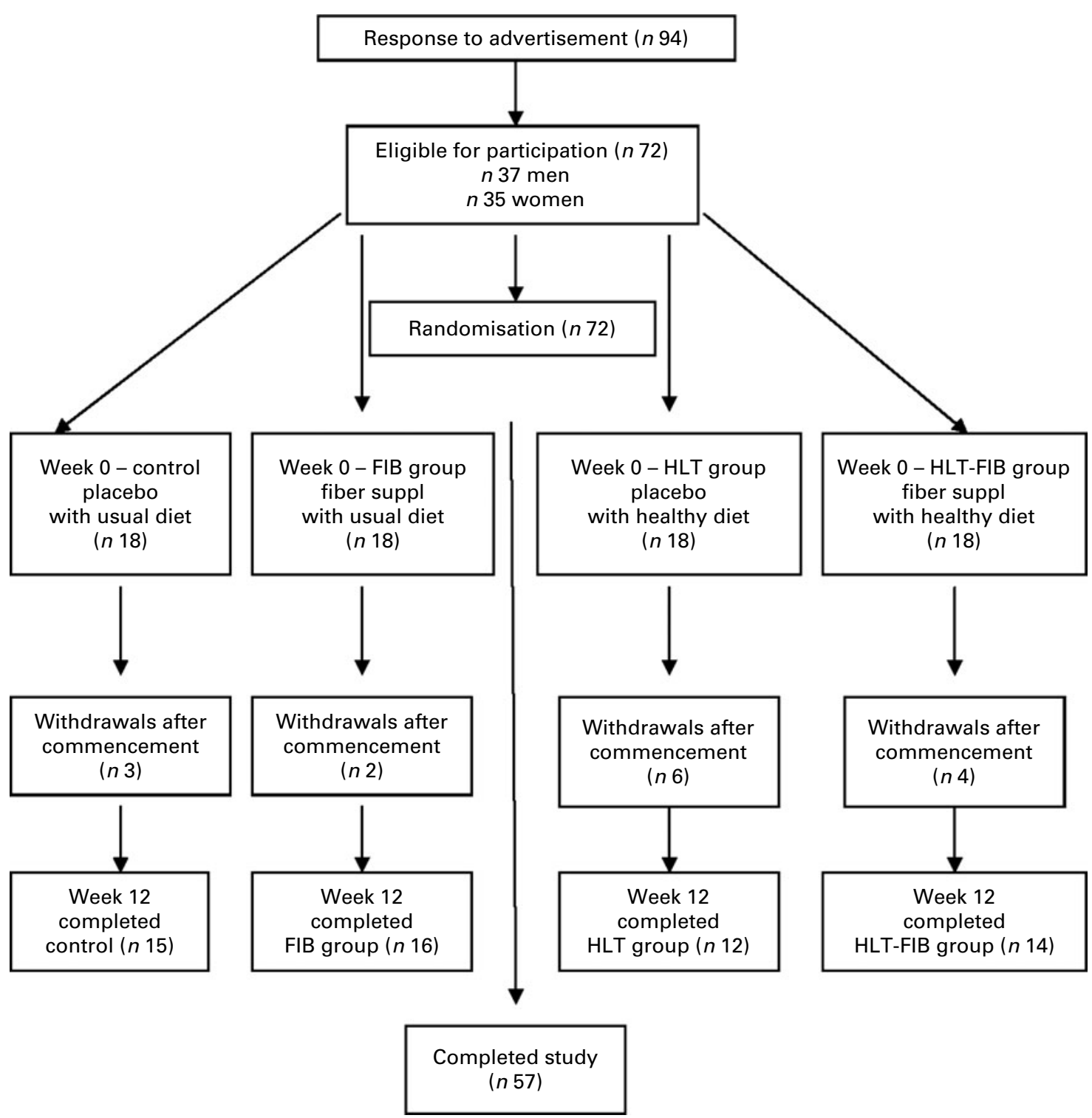

Fig. 1. Details of subject recruitment and withdrawal. FIB, fibre supplement group; HLT, healthy eating group with placebo; HLT-FIB, healthy eating with fibre supplement. 


\section{Measurements of lipids, glucose and insulin}

Serum TAG and TC were measured by enzymatic colorimetric kits (TRACE Scientific Limited, Melbourne, VIC, Australia). Serum HDL-cholesterol (HDL-C) was determined after precipitation of apo B-containing lipoproteins with phosphotungstic acid and $\mathrm{MgCl}_{2}$, the supernatant containing the HDL-C was determined by enzymatic colorimetry (TRACE Scientific Limited). Serum LDL-cholesterol (LDL-C) was determined by using the Friedewald equation. Plasma glucose levels were measured using the Randox glucose GOD-PAP kit (Antrim, UK), according to the manufacturer's instructions. Plasma insulin was measured by an ELISA kit (Dako Diagnostic, Kakkyoyama, Japan).

\section{Statistical analysis}

The sample size of the study is based on the predicted $10 \%$ reduction in TC, LDL-cholesterol, TAG, body weight and total body fat content after intervention. A sample size of at least subjects/group will provide sufficient power $(>90 \%)$ to detect an estimated within and betweengroup effect size of 0.56 at a $5 \%$ significance level. Recruiting a total of seventy-two subjects ( $n$ 18) will accommodate for $20 \%$ dropouts. Statistical analysis was undertaken using SPSS 11 for Windows (SPSS Inc., Chicago, IL, USA). Data were expressed as means with their standard errors and assessed for normality to ensure that the assumptions of the analysis are met. The data were analysed using General Linear Models with baseline value covariates. If significant between-groups effects were present, post hoc comparisons between the treatment groups were made using the Least Significant Difference method. Statistical significance was considered at $P<0 \cdot 05$.

The present clinical trial was registered with the Australian New Zealand Clinical Trials Registry. The registration number is ACTRN12609000540213 and trial web address is https://www.anzctr.org.au/ACTRN12609000540213.aspx

\section{Results}

Subjects

Seventy-two individuals were randomly assigned to one of the four test groups: control, FIB, HLT or HLT-FIB for 12 weeks. Fifteen participants withdrew from the study due to unrelated illness, work commitments, poor compliance and personal reasons. Fifty-seven participants (twenty-five males and thirty-two females) completed the 12-week study (control: $n$ 15; FIB: $n$ 16; HLT: $n$ 12; HLT-FIB: $n$ 14) as shown in Fig. 1. Subject characteristics in the four treatment arms at baseline were not significantly different (Table 1). Although there were some reports of minor bloating initially, all subjects tolerated the supplements well with no adverse effects reported.

\section{Diet analysis}

The self-reported food and drink intake at baseline, 6 and 12 weeks are shown in Table 2. There were no significant differences in total energy, carbohydrate, protein, total fat, saturated fat, monounsaturated fat, polyunsaturated fat and fibre between the four groups at baseline.

The total energy intake in the HLT-FIB group was lower ( $P=0.031$ ) by $13 \%$ compared with the control group at 12 weeks. Fibre intake was higher $(P<0.001)$ in the FIB group by $36 \mathrm{~g}$, higher $(P<0.001)$ in the HLT group by $11 \mathrm{~g}$ and higher $(P<0.001)$ in the HLT-FIB group by $39 \mathrm{~g}$ at week 12 compared with the control group. Fibre intake in the FIB and HLT-FIB groups was also higher $(P<0 \cdot 001)$ than the HLT group by 25 and $28 \mathrm{~g}$, respectively, at week 12. The HLT-FIB group intake was also higher $(P=0.048)$ by $3.7 \mathrm{~g}$ than the FIB group at week 12 .

Table 1. Subject characteristics at baseline*

(Mean values with their standard errors)

\begin{tabular}{|c|c|c|c|c|c|c|c|c|}
\hline \multirow[b]{2}{*}{ Characteristics } & \multicolumn{2}{|c|}{ Control ( $n$ 15) } & \multicolumn{2}{|c|}{ FIB $(n 16)$} & \multicolumn{2}{|c|}{ HLT $(n$ 12) } & \multicolumn{2}{|c|}{$\begin{array}{l}\mathrm{HLT}-\mathrm{FIB} \\
(n 14)\end{array}$} \\
\hline & Mean & SEM & Mean & $\overline{\text { SEM }}$ & Mean & $\overline{\text { SEM }}$ & Mean & SEM \\
\hline Age (years) & $44 \cdot 8$ & 1.6 & $41 \cdot 3$ & $2 \cdot 3$ & $40 \cdot 8$ & 3.0 & 44.9 & $2 \cdot 9$ \\
\hline $\mathrm{Wt}(\mathrm{kg})$ & 97.8 & $3 \cdot 2$ & 101.6 & 3.0 & 101.9 & 4.6 & 98.5 & 3.1 \\
\hline $\operatorname{BMI}\left(\mathrm{kg} / \mathrm{m}^{2}\right)$ & 33.7 & $1 \cdot 0$ & 34.0 & 0.9 & $36 \cdot 1$ & 1.5 & $34 \cdot 2$ & $1 \cdot 2$ \\
\hline Waist circumference $(\mathrm{cm})$ & $104 \cdot 8$ & 3.0 & 110.2 & 3.5 & 110.9 & 3.5 & $106 \cdot 3$ & $2 \cdot 9$ \\
\hline Waist:hip ratio & 0.9 & $2 \cdot 1$ & 0.9 & $2 \cdot 8$ & 0.8 & $2 \cdot 7$ & 0.9 & $2 \cdot 1$ \\
\hline Fasting TAG $(\mathrm{mmol} / \mathrm{l})$ & $2 \cdot 0$ & 0.3 & 1.5 & 0.2 & $1 \cdot 7$ & 0.3 & 1.7 & 0.2 \\
\hline Fasting cholesterol $(\mathrm{mmol} / \mathrm{l})$ & $6 \cdot 1$ & 0.3 & $5 \cdot 9$ & 0.3 & $6 \cdot 3$ & 0.2 & $6 \cdot 3$ & 0.3 \\
\hline Fasting HDL-cholesterol (mmol/l) & 1.3 & 0.1 & 1.3 & 0.1 & $1 \cdot 3$ & 0.1 & 1.3 & 0.2 \\
\hline Fasting LDL-cholesterol (mmol/l) & 3.9 & 0.3 & 4.0 & 0.3 & $4 \cdot 2$ & 0.2 & $4 \cdot 2$ & 0.3 \\
\hline Fasting glucose $(\mathrm{mmol} / \mathrm{l})$ & $5 \cdot 6$ & 0.2 & 6.5 & 0.2 & $5 \cdot 3$ & 0.2 & $6 \cdot 0$ & 0.2 \\
\hline Fasting insulin $(\mu \mathrm{Ul} / \mathrm{ml})$ & $18 \cdot 2$ & 1.8 & $19 \cdot 4$ & $1 . \overline{7}$ & $20 \cdot 4$ & 2.0 & $20 \cdot 4$ & 2.5 \\
\hline
\end{tabular}

FIB, fibre supplement group; HLT, healthy eating group with placebo; HLT-FIB, healthy eating group with fibre supplement.

* Mean values were not significantly different between subject characteristics at screening. 
Table 2. Reported dietary intake data assessed by weighed food records $†$ (Daily mean values with their standard errors, $n$ 57)

\begin{tabular}{|c|c|c|c|c|c|c|}
\hline & \multicolumn{2}{|c|}{ Baseline } & \multicolumn{2}{|c|}{ Week 6} & \multicolumn{2}{|c|}{ Week 12} \\
\hline & Mean & SEM & Mean & SEM & Mean & SEM \\
\hline \multicolumn{7}{|l|}{ Total El } \\
\hline Control & $8054 \cdot 1$ & 428.4 & 8325 & $629 \cdot 2$ & $8165 \cdot 7^{a}$ & 509.2 \\
\hline FIB & 8375.9 & $467 \cdot 1$ & 7817.4 & 359.6 & $7756 \cdot 8^{\mathrm{a}, \mathrm{b}}$ & 369.2 \\
\hline HLT & $8266 \cdot 3$ & 479.2 & $7807 \cdot 6$ & $422 \cdot 7$ & $7255 \cdot 4^{\mathrm{a}, \mathrm{b}}$ & 325.4 \\
\hline HLT-FIB & $8750 \cdot 6$ & 348.8 & $8154 \cdot 4$ & 602.6 & $7121 \cdot 6^{\mathrm{b} *}$ & 298.5 \\
\hline \multicolumn{7}{|c|}{ Carbohydrate (\% of EI) } \\
\hline Control & $43 \cdot 8$ & $2 \cdot 4$ & 43.8 & 2.4 & 44.5 & $2 \cdot 2$ \\
\hline FIB & $45 \cdot 9$ & $2 \cdot 0$ & $45 \cdot 5$ & 1.5 & $46 \cdot 3$ & $2 \cdot 1$ \\
\hline HLT & $45 \cdot 8$ & $1 \cdot 7$ & $47 \cdot 1$ & 1.7 & 49.9 & $2 \cdot 1$ \\
\hline HLT-FIB & $43 \cdot 6$ & $1 \cdot 8$ & $45 \cdot 3$ & 1.0 & $50 \cdot 3^{*}$ & 1.4 \\
\hline \multicolumn{7}{|c|}{ Protein Intake (\% of El) } \\
\hline Control & $18 \cdot 3$ & $1 \cdot 3$ & $18 \cdot 2$ & 1.2 & $18 \cdot 2$ & 1.0 \\
\hline FIB & $17 \cdot 7$ & $1 \cdot 1$ & 18.5 & 1.0 & $19 \cdot 3$ & $1 \cdot 1$ \\
\hline HLT & $17 \cdot 4$ & $1 \cdot 1$ & $19 \cdot 3$ & 1.1 & $21 \cdot 8^{*}$ & $1 \cdot 2$ \\
\hline HLT-FIB & $19 \cdot 8$ & $1 \cdot 2$ & 18.5 & 0.6 & 21.6 & 1.0 \\
\hline \multicolumn{7}{|c|}{ Fat intake (\% of El) } \\
\hline Control & $37 \cdot 8$ & 1.9 & $37.8^{\mathrm{a}}$ & $2 \cdot 1$ & $37.1^{\mathrm{a}}$ & $2 \cdot 1$ \\
\hline FIB & $36 \cdot 2$ & 1.5 & $35 \cdot 9^{a}$ & 0.9 & $34 \cdot 3^{a}$ & 1.7 \\
\hline HLT & $36 \cdot 6$ & 1.6 & $32 \cdot 3^{\mathrm{b} *}$ & 1.4 & $28 \cdot 2^{b *}$ & $1 \cdot 1$ \\
\hline HLT-FIB & $36 \cdot 5$ & $1 \cdot 8$ & $33 \cdot 1^{\mathrm{b}}$ & 1.7 & $28.0^{\mathrm{b} *}$ & $1 \cdot 3$ \\
\hline \multicolumn{7}{|c|}{ SFA intake ( $\%$ of EI) } \\
\hline Control & 14.4 & $1 \cdot 2$ & $17 \cdot 8$ & 1.3 & $19 \cdot 7^{a *}$ & 1.9 \\
\hline FIB & $14 \cdot 6$ & 1.0 & $17 \cdot 0$ & 0.8 & $17 \cdot 4^{\mathrm{a}, \mathrm{b}}$ & $1 \cdot 1$ \\
\hline HLT & 12.4 & 0.8 & $16 \cdot 1^{*}$ & 1.0 & $14 \cdot 6^{\mathrm{b} *}$ & $1 \cdot 1$ \\
\hline HLT-FIB & $15 \cdot 6$ & 1.0 & $16 \cdot 8$ & 1.4 & $14 \cdot 5^{\mathrm{b}}$ & 1.0 \\
\hline \multicolumn{7}{|c|}{ MUFA intake (\% of El) } \\
\hline Control & 14.2 & 0.8 & $13 \cdot 7^{a}$ & $1 \cdot 1$ & $11.4^{\mathrm{a} *}$ & 1.0 \\
\hline FIB & $14 \cdot 2$ & 0.7 & $13 \cdot 0$ & $0.8^{\mathrm{a}}$ & $11 \cdot 3^{\mathrm{a}, \mathrm{b} *}$ & 0.7 \\
\hline HLT & $14 . \overline{5}$ & 0.6 & $10 \cdot 4^{\mathrm{b} *}$ & 1.0 & $9 \cdot 0^{\mathrm{b} *}$ & 0.6 \\
\hline HLT-FIB & $13 \cdot 4$ & 1.0 & $10 \cdot 9^{\mathrm{a}, \mathrm{b} *}$ & 0.7 & $9 \cdot 1^{\mathrm{a}, \mathrm{b} *}$ & 0.7 \\
\hline \multicolumn{7}{|c|}{ PUFA intake (\% of EI) } \\
\hline Control & $9 \cdot 2$ & $1 \cdot 2$ & $6 \cdot 3^{*}$ & 0.6 & $6 \cdot 0 a^{*}$ & 0.5 \\
\hline FIB & 7.5 & 0.7 & $5 \cdot 9^{*}$ & 0.3 & $5 \cdot 7^{\mathrm{a}, \mathrm{b} *}$ & 0.5 \\
\hline HLT & $9 \cdot 8$ & 1.3 & $5 \cdot 9^{\star}$ & 0.6 & $4 \cdot 6^{\mathrm{b} *}$ & 0.4 \\
\hline HLT-FIB & $8 \cdot 1$ & 0.7 & $5 \cdot 4^{*}$ & 0.4 & $4 \cdot 4^{\mathrm{b} *}$ & 0.4 \\
\hline \multicolumn{7}{|c|}{ Fibre intake (g) } \\
\hline Control & $23 \cdot 7$ & $1 \cdot 2$ & $19 \cdot 7^{a *}$ & 0.6 & $19 \cdot 7^{a *}$ & 0.6 \\
\hline FIB & 23.9 & 1.0 & $53 \cdot 1^{\mathrm{b} *}$ & $2 \cdot 0$ & $55 \cdot 3^{\mathrm{c} *}$ & 1.9 \\
\hline HLT & 24.0 & 1.0 & $31.6^{\mathrm{c}}$ & $1 \cdot 2$ & $30 \cdot 7^{b}$ & 0.6 \\
\hline HLT-FIB & $23 \cdot 0$ & 0.8 & $57.5^{\mathrm{d} *}$ & 1.5 & $59 \cdot 0^{\mathrm{d} *}$ & 1.4 \\
\hline
\end{tabular}

EI, energy intake; FIB, fibre supplement group; HLT, healthy eating group with placebo; HLT-FIB, healthy eating group with fibre supplement.

a,b,c,d Mean values with unlike superscript letters were significantly different between groups at 6 and 12 weeks $(P<0.05)$.

* Mean values were statistically significant from baseline $(P<0.05)$.

†The nutritional data recorded in 3-d food diaries at baseline, weeks 6 and 12 .

Total fat intake was lower $(P<0 \cdot 001)$ in the HLT and HLT-FIB groups by 6 and $15 \%$, respectively, at week 6 and both by $24 \%$ at week 12 compared with the control group. When compared with the FIB group, total fat intake was lower $(P<0.001$ and $P=0.020$, respectively) in the HLT and HLT-FIB groups by 10 and $8 \%$, respectively, at week 6 and lower $(P<0.001)$ in both groups by $18 \%$ at week 12. Saturated fat intake was increased $(P<0.05)$ in the control group at 12 weeks compared with baseline. The HLT group also registered an increase $(P<0.05)$ in saturated fat at 12 weeks compared with baseline. Saturated fat intake was reduced $(P=0.029)$ by $26 \%$ in the HLT group and reduced $(P=0.006)$ by $26 \%$ in the HLT-FIB group compared with the control group at week 12 .
Polyunsaturated fat intake was also lower $(P=0 \cdot 018)$ in the HLT group by $23 \%$ and reduced $(P=0.025)$ in the HLT-FIB group by $27 \%$ compared with the control group at week 12. Monounsaturated fat intake in the HLT group was lower $(P=0 \cdot 008)$ by $24 \%$ at week 6 and lower $(P=0.047)$ by $20 \%$ at week 12 compared with the control group.

\section{Body composition}

There were no significant within-group changes in body weight, BMI and \% body fat in the four groups as shown in Fig. 2. When making between-group comparisons, body weight in the FIB, HLT and HLT-FIB groups was lower $(P=0.007, P=0.006$ and $P<0.001$, respectively) 

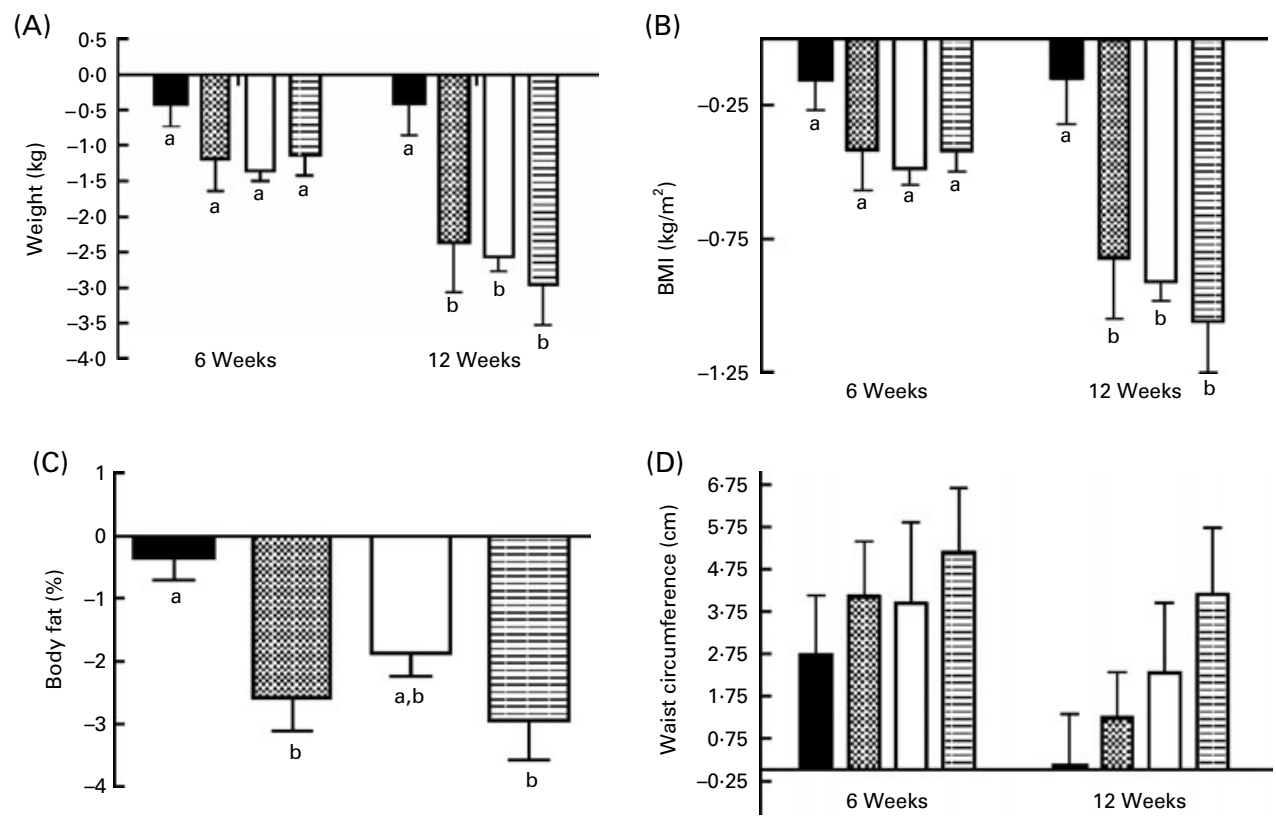

Fig. 2. Changes in body weight (A); BMI (B); \% body fat (C) and waist circumference (D). The changes in body weight, BMI and waist circumference from baseline to 6 and 12 weeks in control, fibre supplement group (FIB), healthy eating group with placebo (HLT) or healthy eating group with fibre supplement (HLT-FIB) groups. The change in percentage body fat from baseline to 12 weeks in control, FIB, HLT or HLT-FIB groups. Data are means with their standard errors. ${ }^{*}$ Mean values were significantly different from baseline $(P<0.05)$. ${ }^{\mathrm{a}, \mathrm{b}}$ Mean values with unlike letters were significantly different between groups $(P<0.05)$. $\mathbf{\square}$, Control; $\boldsymbol{\pi}, \mathrm{FIB} ; \square ; \mathrm{HLT} ;$ 回, HLT-FIB.

compared with the control group at week 12 (Fig. 2(a)). BMI in the FIB, HLT and HLT-FIB groups was also lower ( $P=0.010, \quad P=0.011$ and $P<0.001$, respectively) compared with the control group at week 12 (Fig. 2(b)). Dual-energy X-ray absorptiometry results revealed that the percentage of total body fat in the FIB and HLT-FIB groups was lower $(P=0.002$ and $P<0.001$, respectively) compared with the control group at 12 weeks (Fig. 2(c)). There were no waist circumference differences within or between groups (Fig. 2(d)).

\section{Fasting blood lipids}

Serum TAG levels decreased in the HLT and HLT-FIB groups at 6 and 12 weeks compared with baseline levels (Fig. 3). TAG levels reduced in the HLT-FIB group by $27 \%$ at 6 weeks $(1.27 \mathrm{mmol} / \mathrm{l}, P=0.044)$ and by $33 \%$ at 12 weeks ( $1.17 \mathrm{mmol} / \mathrm{l}, \quad P=0.016)$ compared with baseline levels $(1.75 \mathrm{mmol} / \mathrm{l})$. Between-group comparisons revealed that the TAG levels of the HLT and HLT-FIB groups were both reduced ( $P=0.008$ and 0.003 , respectively) by $38 \%$ (both $1.27 \mathrm{mmol} / \mathrm{l})$, at week 6 and both reduced $(P=0.001$ and $P<0.001$, respectively) by $46 \%(1.16$ and $1.17 \mathrm{mmol} / \mathrm{l}$, respectively) at week 12 compared with the control group $(2.05 \mathrm{mmol} / \mathrm{l}$ at week 6 and $2.16 \mathrm{mmol} / \mathrm{l}$ at week 12). The TAG levels in both the HLT and HLT-FIB groups at week 6 were lower ( $P=0.009$ and 0.004 , respectively) than the FIB group and also lower $(P=0.005$ and 0.003 , respectively) than the FIB group at week 12. A comparison of the changes in TAG levels in all groups is shown in Fig. 3.
There were between- and within-group differences in fasting TC levels in the participants over the 12-week intervention period (Fig. 4(a)). The TC levels decreased from baseline in the HLT $(6.28 \mathrm{mmol} / 1, P=0.002)$ and HLT-FIB ( $6.34 \mathrm{mmol} / \mathrm{l}, P=0.014)$ groups both by $17 \%(5.22$ and $5 \cdot 29 \mathrm{mmol} / 1$, respectively) at week 6 . TC serum levels also significantly reduced at week 12 in the FIB (5.01 mmol $/ 1, P=0.001)$, HLT $(5.15 \mathrm{mmol} / 1, P=0.001)$ and HLT-FIB $(5.07 \mathrm{mmol} / \mathrm{l}, P=0.003)$ groups by 15,18 and $20 \%$, respectively, from baseline. Comparison of differences between groups revealed that TC levels in the FIB, HLT and HLT-FIB groups were lower by $15 \%$ $(P=0.003), 16 \%(P=0.001)$ and $15 \%(P=0.02)$ at week 6, respectively, and lower by $21 \% \quad(P<0.001), \quad 19 \%$ $(P<0.001)$ and 20\% $(P<0.000)$, respectively, at week 12 compared with the control group. There were no significant differences in TC levels between the FIB, HLT and HLT-FIB groups at weeks 6 and week 12. A comparison of the changes in TC levels in all groups is shown in Fig. 4(a).

Fasting LDL-C levels decreased in the FIB, HLT and HLT-FIB groups from baseline (3.97, 4.16 and $4.19 \mathrm{mmol} / 1$, respectively) at week 6 by $22 \%(3.10 \mathrm{mmol} /$ 1, $\quad P=0.013), \quad 22 \% \quad(3.24 \mathrm{mmol} / 1, \quad P=0.006)$ and $22 \%$ (3.26 mmol/1; $P=0.029$ ), respectively (Fig. 4(b)). Fasting LDL was significantly reduced in the FIB, HLT and HLTFIB groups from baseline at week 12 by $27 \%$ ( $2.88 \mathrm{mmol} / \mathrm{l}, P=0.002), 26 \%(3.07 \mathrm{mmol} / 1, P=0.002)$ and $30 \% \quad(2.93 \mathrm{mmol} / 1, \quad P=0.004)$, respectively. Betweengroup comparisons of LDL levels revealed that the FIB, 


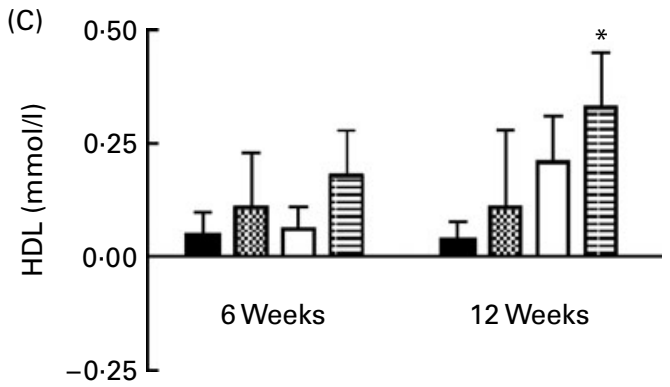

Fig. 4. Changes in total cholesterol (TC) (A); LDL cholesterol (B) and HDL cholesterol (C). The change in TC, LDL and HDL cholesterol from baseline to 6 weeks and 12 weeks in control, fibre supplement group (FIB), healthy eating group with placebo (HLT) or healthy eating group with fibre supplement (HLT-FIB) groups. Data are means with their standard errors. ${ }^{*}$ Mean values were significantly different from baseline $(P<0.05)$. ${ }^{\mathrm{a}, \mathrm{b}}$ Mean values with unlike letters were significantly

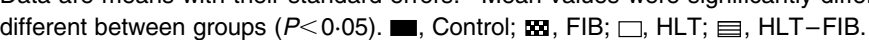
$(6.00 \mathrm{mmol} / \mathrm{l})$ group at week 6 . There were no significant

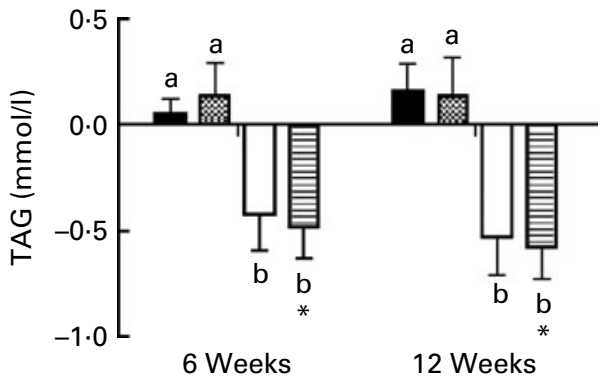

Fig. 3. Changes in fasting serum TAG. The change in TAG from baseline to 6 weeks and 12 weeks in control, fibre supplement group (FIB), healthy eating group with placebo (HLT) or healthy eating group with fibre supplement (HLT-FIB) groups. Data are means with their standard errors. * Mean values were significantly different from baseline $(P<0.05)$. ${ }^{\mathrm{a}, \mathrm{b}}$ Mean values with unlike letters were significantly different between groups $(P<0.05)$.

$\boldsymbol{\square}$, Control; $\boldsymbol{\boldsymbol { \alpha }}, \mathrm{FIB} ; \square, \mathrm{HLT}$; 鸟, HLT-FIB.

HLT and HLT-FIB groups had lower LDL levels by $23 \%$ $(P<0.001), 18 \%(P=0.005)$ and $17 \%(P<0 \cdot 001)$, respectively, compared with the control group at week 6 . The LDL level was lower $(P<0 \cdot 001)$ after 12 weeks of intervention in the FIB, HLT and HLT-FIB groups by 29, 24 and $28 \%$, respectively, compared with the control group $(4.05 \mathrm{mmol} / \mathrm{l})$. A comparison of serum LDL-C changes in all groups is shown in Fig. 4(b).

The HDL-C levels increased significantly $(P=0.02)$ by $21 \%$ in the HLT-FIB group at week $12(1.60 \mathrm{mmol} / \mathrm{l})$ compared with baseline $(1.27 \mathrm{mmol} / \mathrm{l})$. Between-group comparisons revealed no significant differences in HDL levels as shown in Fig. 4(c).

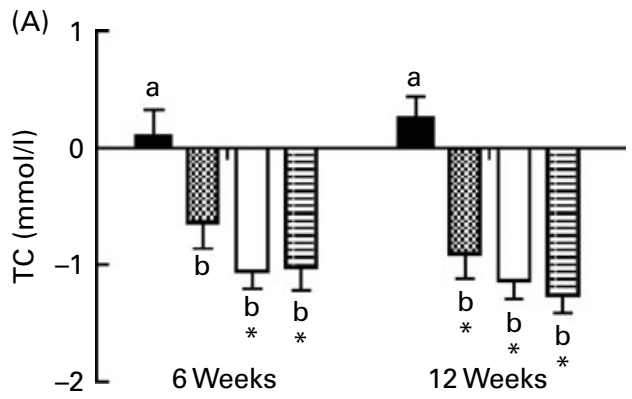

\section{Fasting glucose and insulin}

Within-group comparisons revealed that fasting blood glucose levels were not significantly changed in any treatment group compared with baseline or week 6 as shown in Fig. 5(a). However, when comparing between-group effects, glucose levels were $14 \%(5.16 \mathrm{mmol} / 1, P=0.03)$ lower in the HLT group compared with the control between-group differences at week 12 .

Fasting insulin levels decreased at week 12 in the HLT group by $24 \%(15.62 \mu \mathrm{UI} / \mathrm{ml}, P=0.03)$ and in the HLTFIB group by $33 \%(13.71 \mu \mathrm{UI} / \mathrm{ml}, \quad P=0 \cdot 01)$ compared with baseline (20.44 and $20.43 \mu \mathrm{UI} / \mathrm{ml}$, respectively) (Fig. 5(b)). When making between-group comparisons at week 6, fasting insulin levels were lower in the HLT group by $5 \%(17.33 \mu \mathrm{UI} / \mathrm{ml}, P=0.05)$ and lower in the HLT-FIB group by $8 \%(16.94 \mu \mathrm{UI} / \mathrm{ml}, P=0.02)$ compared with the control group $(18 \cdot 27 \mu \mathrm{UI} / \mathrm{ml})$. When compared with the control group at week $12(19.89 \mu \mathrm{UI} / \mathrm{ml})$, insulin levels decreased in the HLT and HLT-FIB groups by $21 \%(P<0.001)$ and $31 \%(P<0 \cdot 001)$, respectively. When compared with the FIB groups at week $6(19.53 \mu \mathrm{UI} / \mathrm{ml})$, insulin levels were significantly lower in the HLT group by $11 \%(P=0.02)$ and in the HLT-FIB group by $13 \%$ $(P=0 \cdot 01)$. At week 12, insulin levels were lower in the HLT group by $19 \%(P=0.01)$ and in the HLT-FIB group by $28 \% \quad(P<0 \cdot 000)$ compared with the FIB group $(19 \cdot 19 \mu \mathrm{UI} / \mathrm{ml})$. A comparison of insulin changes in all groups is shown in Fig. 5(b)

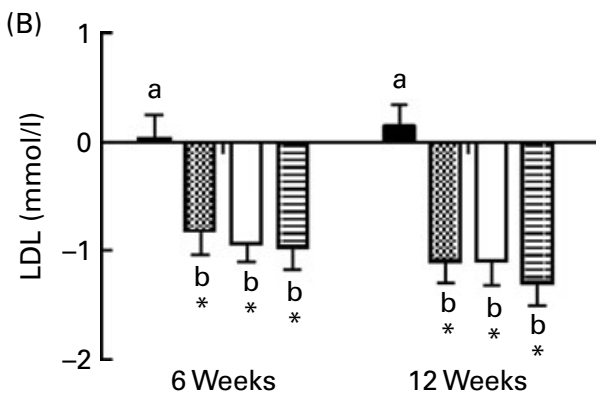



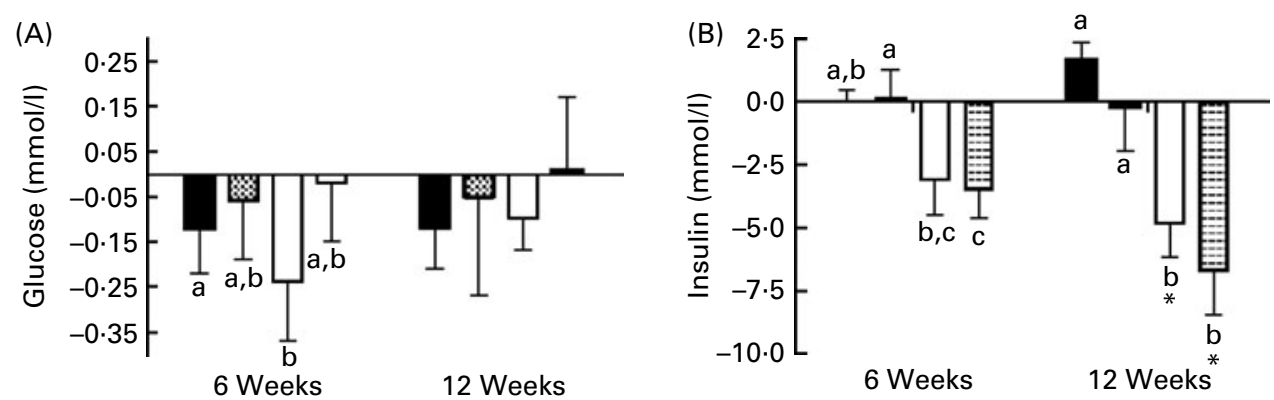

Fig. 5. Changes in fasting serum glucose (A) and insulin (B). The change in glucose and insulin from baseline to 6 weeks and 12 weeks in control, fibre supplement group (FIB), healthy eating group with placebo (HLT) or healthy eating group with fibre supplement (HLT-FIB) groups. Data are means with their standard errors. * Mean values were significantly different from baseline $(P<0.05) .{ }^{\mathrm{a}, \mathrm{b}, \mathrm{c}}$ Mean values with unlike letters were significantly different between groups $(P<0.05)$. $\boldsymbol{\square}$, Control; $\boldsymbol{s}, \mathrm{FIB} ; \square, \mathrm{HLT} ;$ 目, HLT-FIB.

\section{Discussion}

The present study demonstrated that simply adding psyllium fibre supplementation to a normal diet (FIB group, $55 \mathrm{~g} / \mathrm{d}$ ) was sufficient to see improvements in TC and LDL-C as well as body weight, BMI and \% body fat compared with control. Consuming a healthy diet with moderate fibre intake (HLT group, $30 \mathrm{~g}$ ) showed improvements in TC, LDL-C, TAG, insulin, body weight and BMI compared with the control. Consuming both the fibre supplement and the healthy diet (HLT-FIB, 59 g) showed benefits in all of these parameters compared with control with a non-significant trend towards a greater effect on TC, LDL-C, TAG, insulin, body weight, BMI and \% body fat compared with the other intervention groups. Therefore, recommendations for $>30 \mathrm{~g} / \mathrm{d}$ of fibre for overweight or obese individuals, derived from dietary sources in conjunction with psyllium fibre supplementation, may be appropriate to improve metabolic syndrome risk factors in this group.

Many studies have found that increasing fibre intake has resulted in increased satiety, reduced hunger, decreased energy intake and weight loss ${ }^{(3,33)}$. In the present study, we found that body weight and BMI were significantly lower in the FIB, HLT and HLT-FIB groups compared with the control at 12 weeks although the FIB and HLTFIB groups also showed a significant reduction in \% body fat. The reduction in \% body fat in these two groups may indicate that a high fibre intake of over $55 \mathrm{~g}$ from a psyllium supplement, with or without a healthy diet, may influence body composition as well as body weight. The greatest anthropometric changes were found in the HLT-FIB group, which may be partly explained by a significant reduction in reported total energy intake. Epidemiological and cohort evidence indicate that diets rich in fibre are associated with lower body weight ${ }^{(2,6,17)}$. A recent study by Du et al. ${ }^{(13)}$ found an inverse association of total dietary fibre and cereal fibre with weight and waist circumference change in a cohort of 89432 European adults who were studied over an average period of 6.5 years. Interestingly, the present study revealed no differences in waist circumference in any intervention group, which may suggest that a longer time is required to see such observable effects. Howarth $e t a l .{ }^{(3)}$ reviewed twelve intervention studies and found that when energy intake is ad libitum, an increase of $14 \mathrm{~g} / \mathrm{d}$ of fibre over a standard diet resulted in a $10 \%$ decrease in energy intake and a $1.9 \mathrm{~kg}$ decrease in body weight over 3.8 months. However, in two reviews undertaken by Pittler \& Ernst ${ }^{(22,34)}$, they found unconvincing evidence that fibre supplements can reduce body weight. Other intervention studies also reveal no change in body weight ${ }^{(23,35)}$. Fibre's effect on satiety and reduced energy intake may be due to its reduced energy density and increased chewing time ${ }^{(33)}$. Fibre may affect satiety by slowing gastric emptying time, increasing viscosity in the gastrointestinal tract and therefore reducing postprandial glycaemia and insulin secretion ${ }^{(4)}$. Fibre may also influence satiety hormone levels such as ghrelin ${ }^{(36)}$. A review by Pereira \& Ludwig ${ }^{(33)}$ revealed that dietary fibre had beneficial influences on satiety and energy intake in seventeen studies, whereas seven studies reported mixed results and three studies reported no effects. In our previous acute study, $15 \mathrm{~g}$ of dietary fibre did not affect the hunger and satiety in the postprandial period ${ }^{(37)}$. Evidence is inconclusive in relation to the effects of fibre on weight loss, but the present study suggests that larger amounts of fibre $(>30 \mathrm{~g})$ may be required to alter energy balance sufficiently for weight loss and to bring about changes in body composition.

The HLT and the HLT-FIB groups had the greatest impact on lowering serum TAG levels at 6 and 12 weeks compared with the control. Previous studies have shown inconsistent results regarding the effect of dietary fibre on TAG metabolism ${ }^{(9)}$ with most of them indicating no effect $^{(21,27,28)}$. However, in a 6 -week randomised study by Chandalia et $a l^{(24)}$ in subjects with type 2 diabetes, intake of a high-fibre diet with high-soluble fibre content (total fibre intake $50 \mathrm{~g} / \mathrm{d}$, including $25 \mathrm{~g} / \mathrm{d}$ soluble fibre) lowered TAG concentrations by $10 \cdot 2 \%{ }^{(24)}$. Although mechanisms are not fully understood, the apparent 
hypotriacylglycerolaemic effects of psyllium and other soluble fibres may be related to a delay in the absorption of TAG and carbohydrates from the small intestine ${ }^{(38)}$. In the present study, there was no observed reduction in serum TAG levels in the FIB group despite a fibre intake similar to the HLT-FIB group of over $55 \mathrm{~g}$ and greater than the HLT group fibre intake of $31 \mathrm{~g}$. Wu et al. ${ }^{(9)}$ found that the highest quintile of fibre consumption (total, non-viscous and pectin) was associated with the lowest TAG levels. The psyllium supplement consumed in the present study may not be as effective as the multiple sources of fibre from consuming non-viscous fibres and pectin from wholegrains, fruit and vegetables.

Consuming a fibre supplement (FIB) or consuming a healthy diet (HLT) both reduced serum TC and LDL levels, but the combination of both, in the HLT-FIB group, produced the greatest reduction, although there was no significant difference between the FIB, HLT and HLT-FIB groups. This indicates that fibre intake at $30 \mathrm{~g}$ or over was sufficient to reduce serum TC and LDL-C whether from consuming a healthy diet, a fibre supplement alone or both combined. Many clinical and animal trials have demonstrated the hypocholesterolaemic effects of soluble fibres ${ }^{(17,20,21,27,39,40)}$. A meta-analysis by Brown et $a .^{(21)}$ found that soluble fibre from oats, psyllium, pectin and guar gum each reduced TC significantly. Psyllium's hypocholesterolaemic properties have been well researched, with studies indicating that the effects do not depend on the amount of fat or cholesterol in the $\operatorname{diet}^{(21,29,30,41,42)}$. Brown et al. ${ }^{(21)}$ concluded that each gram of fibre from psyllium reduced TC and LDL-C concentrations by -0.028 and $-0.029 \mathrm{mmol} / 1$, respectively. Soluble fibre is thought to entrap bile acids, resulting in a greater faecal loss ${ }^{(30)}$, and is thought to reduce cholesterol absorption and bile acid reabsorption by disrupting the intraluminal formation of micelles ${ }^{(43,44)}$.

There was no difference in HDL-C between groups during the study. However, HDL-C increased in the HLT-FIB group from baseline during the study and it was $19 \%$ higher at the end of the study. Studies have shown that dietary fibre does not affect HDL-C ${ }^{(45)}$. Therefore, the HDL-C increase in the HLT-FIB group may have been due to changes in other dietary components during the study or related weight loss in these subjects. Weight loss has been associated with increased HDL- $\mathrm{C}^{(46)}$. Interestingly, Jenkins et $a l .{ }^{(47)}$ reported that HDL-C was significantly decreased after 4 weeks of psyllium supplementation in weight-stable healthy subjects. Therefore, the present study emphasises the importance of accompanying weight loss with increased dietary fibre to prevent the reduction in HDL-C.

Previous studies have reported an association between high fibre consumption and reduced fasting insulin ${ }^{(20)}$. Ludwig $^{(5)}$ found that, when adjusted for BMI, higher levels of insulin were found in individuals who consumed the least fibre, indicating that a low-fibre diet stimulates a higher insulin response compared with a high-fibre diet. Soluble fibre, such as psyllium, may improve insulin sensitivity by increasing the viscosity of the stomach contents, which slows the digestion and absorption of carbohydrate and other nutrients ${ }^{(31,48)}$. In the present study, insulin levels decreased significantly in the HLT and HLT-FIB groups compared with the control and FIB groups at 12 weeks. Simply consuming additional fibre as a psyllium supplement was not sufficient to influence insulin levels, even at $55 \mathrm{~g}$ fibre/d. The consumption of a healthy diet appeared to have more of an influence on insulin levels which could be related to other factors in that diet.

In the present study, the reduction in body weight in the FIB, HLT and HLT-FIB groups and the lowered TAG and insulin in the HLT-FIB and HLT groups may also have been influenced by dietary factors such as a reduced fat intake $^{(49,50)}$. The two groups that had the reduction in serum TAG levels (HLT-FIB and HLT) also recorded a reduction in reported \% energy intake from total dietary fat and saturated fat and an increase in PUFA compared with the control group (Table 2). However, the control group showed a significant increase in \% energy from saturated fat compared with baseline, which may partly account for the decrease in TC and LDL in the FIB, HLT and HLT-FIB groups. The amount and type of fat consumed in the diet can influence serum TAG levels ${ }^{(15)}$ and serum cholesterol levels ${ }^{(29)}$, which may have had some influence in the present study. The beneficial changes in TC, LDL-C, TAG and insulin levels in the intervention groups may also be related to reduced body weight and reduced adiposity (Fig. 2) as a result of energy restriction. Non-significant energy restriction occurred in the FIB and HLT groups and significantly in the HLT-FIB group relative to control. Numerous studies have reported the improvements in biomarkers with energy restriction and weight loss $(51-55)$.

Most of the subjects in the present study had a waist circumference greater than that specified by the ATP III Clinical Identification of Metabolic Syndrome ${ }^{(1)}$ and they had at least one or two other metabolic syndrome risk factors or were borderline candidates. Many in this group will eventually progress to establish the metabolic syndrome if their obesity continues and lifestyle factors remain unchanged. In the present study, if consumption of a high-fibre diet (>50 g/d) over 12 weeks resulted in gradual improvements leading to significant reductions in weight, $\%$ body fat, lipids and insulin when compared to a control, then intake over a longer period may provide greater results and improved outcomes for overweight and obese individuals. Given that the psyllium supplement taken in conjunction with a healthy diet was well tolerated, investigations into the long-term intake of $>50 \mathrm{~g}$ fibre/d in this group should be pursued further.

In the present study, the comparison between a normal diet (control), a normal diet with a psyllium fibre supplement (FIB), a healthy diet (HLT) and a healthy diet 
with psyllium fibre supplement (HLT-FIB) helped to demonstrate the amount of fibre that is sufficient to improve some of the metabolic syndrome risk factors in overweight and obese individuals as well as the effectiveness of psyllium supplementation and healthy dietary practices. Since the healthy diet and psyllium fibre supplement (HLT-FIB) produced the best results in relation to improving metabolic syndrome risk factors compared with the other groups, a similar dietary regimen could be prescribed to overweight and obese individuals, a population susceptible to the metabolic syndrome, CVD and type 2 diabetes. Therefore, further investigations into increasing dietary recommendations for dietary fibre intake $>30 \mathrm{~g} / \mathrm{d}$ for overweight or obese individuals from dietary sources in conjunction with fibre supplementation should be undertaken.

\section{Acknowledgements}

This trial was partially funded by the ATN Centre for Metabolic Fitness. A. K. coordinated the trial, conducted data collection, statistical analysis and had input into the manuscript. The authors declare that they have no conflicts of interest. S. P. conceived of and designed the study, wrote the manuscript, and supervised the study and the statistical analysis. S. D. had input into the statistical analysis. C. B. and V. E. had input into the writing of the manuscript.

\section{References}

1. Grundy S, Brewer H, Cleeman J, et al. (2004) Definition of metabolic syndrome: report of the National Heart, Lung and Blood Institute/American Heart Association Conference on scientific issues related to definition. Circulation 109, $433-438$.

2. Slavin JL (2005) Dietary fiber and body weight. Nutrition $\mathbf{2 1}$, 411-418.

3. Howarth NC, Saltzman E \& Roberts SB (2001) Dietary fiber and weight regulation. Nutr Rev 59, 129.

4. Lairon D (2007) Dietary fiber and control of body weight. Nutr Metab Cardiovasc Dis 17, 1-5.

5. Ludwig DS (1999) Dietary fiber, weight gain, and cardiovascular disease risk factors in young adults. JAMA 282, 1539.

6. Kromhout D, Bloemberg B, Seidell JC, et al. (2001) Physical activity and dietary fiber determine population body fat levels: the Seven Countries Study. Int J Obes 25, 301-306.

7. National Health and Medical Research Council (2005) National Health and Medical Research Council Nutrient Reference Values for Australia and New Zealand. Canberra: Commonwealth of Australia.

8. American Heart Association (2009) Fiber. http://www. americanheart.org/presenter.jhtml?identifier $=4574$ (updated 2 October 2009).

9. Wu H, Dwyer KM, Fan Z, et al. (2003) Dietary fiber and progression of atherosclerosis: the Los Angeles Atherosclerosis Study. Am J Clin Nutr 78, 1085-1091.

10. State of Victoria. Fibre in Food. Melbourne; 2008. http:// www.betterhealth.vic.gov.au/BHCV2/bhcarticles.nsf/pages/ Fibre_in_food (updated 2 October 2009).

11. National Health and Medical Research Council (2003) National Health and Medical Research Council Dietary
Guidelines for Australian Adults. Canberra: Commonwealth of Australia.

12. US Department of Health and Human Services, US Department of Agriculture (2005) Dietary Guidelines for Americans. Washington, DC: US Government Printing Office.

13. Du H, van der A DL, Boshuizen HC, et al. (2010) Dietary fiber and subsequent changes in body weight and waist circumference in European men and women. Am J Clin Nutr 91, 329-336.

14. Newby P, Maras J, Bakun P, et al. (2007) Intake of whole grains, refined grains, and cereal fiber measured with 7-d diet records and associations with risk factors for chronic disease. Am J Clin Nutr 86, 1745-1753.

15. Lairon D (2008) Macronutrient intake and modulation on chylomicron production and clearance. Atheroscler Suppl $\mathbf{9}$, 45-48.

16. Kan H, Stevens J, Heiss G, et al. (2007) Dietary fiber intake and retinal vascular caliber in the Atherosclerosis Risk in Communities Study. Am J Clin Nutr 86, 1626-1632.

17. Lairon D, Arnault N, Bertrais S, et al. (2005) Dietary fiber intake and risk factors for cardiovascular disease in French adults. Am J Clin Nutr 82, 1185-1194.

18. Venn BJ \& Mann JI (2004) Cereal grains, legumes and diabetes. Eur J Clin Nutr 58, 1443-1461.

19. Weickert MO \& Pfeiffer AFH (2008) Metabolic effects of dietary fiber consumption and prevention of diabetes. $J$ Nutr 138, 439-442.

20. McKeown NM, Meigs JB, Liu S, et al. (2002) Whole-grain intake is favorably associated with metabolic risk factors for type 2 diabetes and cardiovascular disease in the Framingham Offspring Study. Am J Clin Nutr 76, 390-398.

21. Brown L, Rosner B, Willett WW, et al. (1999) Cholesterollowering effects of dietary fiber: a meta-analysis. Am J Clin Nutr 69, 30-42.

22. Pittler MH \& Ernst E (2001) Guar gum for body weight reduction: meta-analysis of randomized trials. Am J Med 110, 724-730.

23. Howarth NC, Saltzman E, McCrory MA, et al. (2003) Fermentable and nonfermentable fiber supplements did not alter hunger, satiety or body weight in a pilot study of men and women consuming self-selected diets. J Nutr 133, 3141-3144.

24. Chandalia M, Garg A, Lutjohann D, et al. (2000) Beneficial effects of high dietary fiber intake in patients with type 2 diabetes mellitus. N Engl J Med 342, 1392-1398.

25. Anderson JW, Randles KM, Kendall CWC, et al. (2004) Carbohydrate and fiber recommendations for individuals with diabetes: a quantitative assessment and meta-analysis of the evidence. J Am Coll Nutr 23, 5-17.

26. Hollenbeck CB, Coulston AM \& Reaven GM (1986) To what extent does increased dietary fiber improve glucose and lipid metabolism in patients with noninsulin-dependent diabetes mellitus (NIDDM)? Am J Clin Nutr 43, 15-24.

27. Aller R, de Luis DA, Izaola O, et al. (2004) Effect of soluble fiber intake in lipid and glucose levels in healthy subjects: a randomized clinical trial. Diabetes Res Clin Pract 65, 7-11.

28. Chen J, He J, Wildman RP, et al. (2006) A randomized controlled trial of dietary fiber intake on serum lipids. Eur J Clin Nutr 60, 62-68.

29. Bell LP, Hectorn KJ, Reynolds H, et al. (1990) Cholesterollowering effects of soluble-fiber cereals as part of a prudent diet for patients with mild to moderate hypercholesterolemia. Am J Clin Nutr 52, 1020-1026.

30. Anderson JW, Allgood LD, Turner J, et al. (1999) Effects of psyllium on glucose and serum lipid responses in men with type 2 diabetes and hypercholesterolemia. Am J Clin Nutr 70, 466-473. 
31. Leclere CJ, Champ M, Boillot J, et al. (1994) Role of viscous guar gums in lowering the glycemic response after a solid meal. Am J Clin Nutr 59, 914-921.

32. Birketvedt GS, Aaseth J, Florholmen JR, et al. (2000) Longterm effect of fibre supplement and reduced energy intake on body weight and blood lipids in overweight subjects. Acta Med (Hradec Kralove) 43, 129-132.

33. Pereira MA \& Ludwig DS (2001) Dietary fiber and bodyweight regulation: observations and mechanisms. Pediatr Clin North Am 48, 969-980.

34. Pittler MH \& Ernst E (2004) Dietary supplements for bodyweight reduction: a systematic review. Am J Clin Nutr $\mathbf{7 9}$, $529-536$

35. Rodriguez-Moran M, Guerrero-Romero F \& Lazcano-Burciaga G (1998) Lipid and glucose lowering efficacy of Plantago Psyllium in type II diabetes. J Diabetes Complications 12, 273-278.

36. St-Pierre D, Rabasa-Lhoret R, Lavoie M-E, et al. (2009) Fiber intake predicts ghrelin levels in overweight and obese postmenopausal women. Eur J Endocrinol 161, 65-72.

37. Khossousi A, Binns CW, Dhaliwal SS, et al. (2007) The acute effects of psyllium on postprandial lipaemia and thermogenesis in overweight and obese men. Br J Nutr 99, 1068-1075.

38. Ebihara K \& Schneeman BO (1989) Interaction of bile acids phospholipids, cholesterol and triglyceride with dietary fibers in the small intestine of rats. J Nutr 119, 1100-1106.

39. Galisteo M, Sanchez M, Vera R, et al. (2005) A diet supplemented with husks of Plantago ovata reduces the development of endothelial dysfunction, hypertension, and obesity by affecting adiponectin and TNF in obese Zucker rats. J Nutr 135, 2399-2404.

40. Valero R, Lorec A, Paganelli F, et al. (2005) Fasting apoprotein B-48 level and coronary artery disease in a population without frank fasting hypertriglyceridemia. Metabolism $\mathbf{5 4}$, 1442-1447.

41. Garvin JE, Forman DT, Eiseman WR, et al. (1965) Lowering of human serum cholesterol by an oral hydrophilic colloid. Proc Soc Exp Biol Med 120, 744-746.

42. Theuwissen E \& Mensink RP (2008) Water-soluble dietary fibers and cardiovascular disease. Physiol Behav 94, 285-292.

43. Anderson JW, Davidson MH, Blonde L, et al. (2000) Longterm cholesterol-lowering effects of psyllium as an adjunct to diet therapy in the treatment of hypercholesterolemia. Am J Clin Nutr 71, 1433-1438.
44. Anderson JW, Riddell-Mason S, Gustafson NJ, et al. (1992) Cholesterol-lowering effects of psyllium-enriched cereal as an adjunct to a prudent diet in the treatment of mild to moderate hypercholesterolemia. Am J Clin Nutr 56, 93-98.

45. Theuwissen E \& Mensink R (2008) Water-soluble dietary fibers and cardiovascular disease. Physiol Behav 94, 285-292.

46. Poobalan A, Aucott L, Precious E, et al. (2009) Weight loss interventions in young people (18 to 25 year olds): a systematic review. Obes Rev Oct [Epublication ahead of print] 28.

47. Jenkins D, Wolever T, Vidgen E, et al. (1997) Effect of psyllium in hypercholesterolemia at two monounsaturated fatty acid intakes. Am J Clin Nutr 65, 1524-1533.

48. Cameron-Smith D, Collier GR \& O'Dea K (1994) Effect of soluble dietary fibre on the viscosity of gastrointestinal contents and the acute glycaemic response in the rat. Br J Nutr $\mathbf{7 1}$, $563-571$.

49. Lanza E, Schatzkin A, Daston C, et al. (2001) Implementation of a $4-y$, high-fiber, high-fruit-and-vegetable, low-fat dietary intervention: results of dietary changes in the Polyp Prevention Trial. Am J Clin Nutr 74, 387-401.

50. Saquib N, Rock CL, Natarajan L, et al. (2009) Does a healthy diet help weight management among overweight and obese people? Health Educ Behav 36, 518-531.

51. Markovic T, Campbell L, Balasubramanian S, et al. (1998) Beneficial effect on average lipid levels from energy restriction and fat loss in obese individuals with or without type 2 diabetes. Diabetes Care. 21, 695-700.

52. de Castro J, Dias T, Chambel P, et al. (2009) A randomized double-blind study comparing the efficacy and safety of orlistat versus placebo in obese patients with mild to moderate hypercholesterolemia. Rev Port Cardiol 28, 1361-1374.

53. Acharya S, Elci O, Sereika S, et al. (2009) Adherence to a behavioral weight loss treatment program enhances weight loss and improvements in biomarkers. Patient Prefer Adherence 3, 151-160.

54. Abbasi F, Chen Y, Farin H, et al. (2008) Comparison of three treatment approaches to decreasing cardiovascular disease risk in nondiabetic insulin-resistant dyslipidemic subjects. Am J Cardiol 102, 1, 64-69.

55. Raatz S, Torkelson C, Redmon J, et al. (2005) Reduced glycemic index and glycemic load diets do not increase the effects of energy restriction on weight loss and insulin sensitivity in obese men and women. J Nutr. 135, 2387-2391. 